\title{
Editorial: Our vision for Physical Review Research
}

We, the Editors of Physical Review Research, are excited to introduce this fully open access journal covering the entire range of physics and related areas, and offering the peer review, author experience, and quality which researchers have come to expect from a Physical Review journal.

The Physical Review family of journals have a long and distinguished history of serving the physics community by publishing original, novel, and significant papers that, in many cases, have become landmarks in their fields of research. The traditional Physical Review A through $E$, and the recent additions of Physical Review Applied, Fluids, and Materials continue to set the standards for physics dissemination due to the quality of their articles as well as to their rigorous peer-review process.

Recent years have seen a substantial change in the physics publishing landscape in both the content being offered and the way in which it is disseminated. The Physical Review journals are at the forefront of this change and have entrusted us to lead and manage the newest title of the family, Physical Review Research, which will provide a fully open access option for all researchers in the physics and related communities to experience the value and quality that the Physical Review journals have delivered to the scientific community for decades.

Physics has become a truly interdisciplinary and inclusive endeavor. The artificial barriers that separated it from the other sciences are being broken down, and modern physics is an exciting discipline at the intersection of - and bridging between - the natural sciences, engineering, and social sciences. Physical Review Research will be a reflection of this new image. Rather than saying "physics is what physicists do', we articulate our scope in a more expansive manner: "physics is the science in which physicists are interested."

Associated with this change, there is a growing demand for broader access to scientific knowledge, with funding agencies and authors increasingly pursuing fully-open access options that allow for maximum dissemination of research outputs. Within the Physical Review family, Physical Review X provides an open access journal showcasing the most selective papers in pure, applied and interdisciplinary physics. Now, Physical Review Research will complement this title by providing a fully open access title with rigorous peer-review standards and with the same acceptance criteria as Physical Review $A$ through $E$, Physical Review Applied, Physical Review Fluids, and Physical Review Materials. Our goal is to establish a publication that offers the same experience and quality that we value and trust, while increasing authors' choice within the Physical Review portfolio. As such, we welcome authors of quality manuscripts not accepted by Physical Review X or Physical Review Letters to seamlessly transfer their work to be considered for publication in Physical Review Research.

In addition to maximizing reach and readership through open access, by covering the full scope of activities in physics and associated disciplines, we will provide an integrated and cohesive view of the forefront of modern physics for our readers and create an outlet for research that addresses the interdisciplinary challenges that we face in modern society. The first articles that we are now presenting begin to show the diversity of topics that will define the scope of Physical Review Research, including papers in condensed matter physics, material science, optics, quantum information, plasma science, and soft matter. Additionally, the first issue will include papers in astrophysics, atomic and molecular physics, chemical physics, complex systems, particles and fields, or topological physics amongst other subject areas. Going 
forward we will continue publishing novel and significant papers in a broad range of additional forefront topics with a clear connection to physics and related fields.

We are confident that this journal will provide a valuable service to all communities with a connection to physics and hope that all scientists in those communities will find Physical Review Research a natural home, both as authors and readers. We look forward to reading about your exciting new scientific discoveries in Physical Review Research.

Raissa D'Souza

co-Lead Editor

Jian-Wei Pan

co-Lead Editor

Nicola Spaldin

co-Lead Editor

Juan José Liétor-Santos

Managing Editor

Published 9 August 2019

DOI: 10.1103/PhysRevResearch.1.010002 